\title{
Neural correlates of rumination in depression
}

\author{
REBecCA E. COONEY \\ Stanford University, Stanford, California \\ JUTTA JOORMANN \\ University of Miami, Coral Gables, Florida \\ FANNy Eugène \\ Concordia University, Montréal, Québec, Canada \\ AND \\ Emily L. Dennis and Ian H. Gotlib \\ Stanford University, Stanford, California
}

\begin{abstract}
Rumination, or recursive self-focused thinking, has important implications for understanding the development and maintenance of depressive episodes. Rumination is associated with the worsening of negative mood states, greater affective responding to negative material, and increased access to negative memories. The present study was designed to use fMRI to examine neural aspects of rumination in depressed and healthy control individuals. We used a rumination induction task to assess differences in patterns of neural activation during ruminative self-focus as compared with a concrete distraction condition and with a novel abstract distraction condition in 14 participants who were diagnosed with major depressive disorder and 14 healthy control participants. Depressed participants exhibited increased activation in the orbitofrontal cortex, subgenual anterior cingulate, and dorsolateral prefrontal cortex as compared with healthy controls during rumination versus concrete distraction. Neural activity during rumination versus abstract distraction was greater for depressed than for control participants in the amygdala, rostral anterior cingulate/medial prefrontal cortex, dorsolateral prefrontal cortex, posterior cingulate, and parahippocampus. These findings indicate that ruminative self-focus is associated with enhanced recruitment of limbic and medial and dorsolateral prefrontal regions in depression. Supplemental materials for this article may be downloaded from http://cabn.psychonomic-journals.org/content/supplemental.
\end{abstract}

Rumination - a pattern of recursive thinking focused on one's negative mood - is hypothesized to serve as a vulnerability factor in the development and maintenance of depressive episodes. Rumination has been shown to prolong and deepen episodes of depression by perpetuating depressed mood (see Nolen-Hoeksema, Wisco, \& Lyubomirsky, 2008, for a recent review) and to increase the risk of developing subsequent episodes (Roberts, Gilboa, \& Gotlib, 1998). Rumination has also been found to be associated with biases in the processing of emotional material (Joormann, Dkane, \& Gotlib, 2006). Given the consistent association of rumination with depression, it is critical to examine more closely the mechanisms underlying rumination in this disorder. Unlike some cognitive processes (e.g., selective attention), however, the act of rumination is not easily observed and assessed. In fact, only one recent study has examined the association between levels of rumination and brain function during different forms of self-reflection (i.e., hopes and aspirations vs. goals and obligations) in de- pression (Johnson, Nolen-Hoeksema, Mitchell, \& Levin, 2009). Rumination likely involves a broad range of cognitive and affective subprocesses that are associated with activation in diverse brain regions, including attention, self-referential processing, and recall of autobiographical memories. Consequently, it is probable that the process of rumination involves coordinated neural function within a distributed and overlapping network of brain regions subserving these component subprocesses.

Indeed, cognitive theories suggest that depressed people differ from their nondepressed counterparts in precisely these proposed processes of rumination, exhibiting increased attention to negative information, particularly if it is self-referential, and better memory for negative than for positive stimuli (see Gotlib \& Joormann, 2010, for a review). Recent studies have reported increased amygdala responding as depressed individuals attend to and remember negative emotional material (e.g., Hamilton \& Gotlib, 2008). Other work highlights the function of the amygdala

R. E. Cooney, rcooney@stanford.edu 
and associated limbic regions during the sustained processing of negative emotional material. For example, Siegle and colleagues (Siegle, Carter, \& Thase, 2006; Siegle, Steinhauser, Thase, Stenger, \& Carter, 2002) found that selfreported rumination scores were correlated with increased and sustained amygdala activation for negative words in depressed individuals. Self-reported rumination has also been found to be associated with amygdala activation during the up-regulation of negative affect in healthy controls, suggesting that an increased tendency to ruminate, even in nondepressed individuals, exacerbates the neural processing of negative information (Ray et al., 2005). Thus, the relation between amygdala responsivity and emotional activation appears to be a key component of the neural network underlying rumination.

It is likely that rumination also recruits the same brain regions that have been found to be related to representations of the "self." Investigators have implicated the medial prefrontal cortex (MPFC), in addition to limbic structures, in self-referential processing (Ochsner \& Gross, 2005; Schmitz \& Johnson, 2006), self-reflection (Johnson et al., 2006), and appraisals of the self-relevance of stimuli (Fossati et al., 2003). Self-referential processing is also an important component of autobiographical memory (Howe \& Courage, 1997), which has been found to activate a network of regions including the MPFC, frontal cortex, posterior cingulate, hippocampus, and parahippocampus (Maguire, 2001; Svoboda, McKinnon, \& Levine, 2006). Interestingly, dysregulation of the MPFC, and of nearby structures such as the rostral anterior cingulate gyrus (rACC), has been hypothesized to play a role in the development and recurrence of depression (see, e.g., Keedwell, Andrew, Williams, Brammer, \& Phillips, 2005; Lemogne et al., 2009). Thus, involvement of the MPFC in mediating autobiographical or self-referential content, coupled with hyperactivity in limbic structures such as the amygdala and hippocampus, may contribute to the ruminative selffocus that characterizes depressed individuals.

The present study was designed to identify and elucidate differences between clinically depressed and neverdisordered participants in patterns of neural activation and self-reported mood ratings associated with ruminative thought. Examinations of the form and content of rumination suggest that ruminative thoughts are past oriented and center on unresolved goals associated with the self (Segerstrom, Tsao, Alden, \& Craske, 2000; Watkins, Moulds, $\&$ Mackintosh, 2005). Moreover, rumination may be less overtly verbal and more passive than are other negative thinking states, such as worry (Papageorgiou \& Wells, 1999). To allow for the assessment of neural functioning during a ruminative state, we designed the rumination induction (RUM IND) task using written statements adapted from Nolen-Hoeksema and Morrow $(1991,1993)$. These statements have been used in several studies to successfully induce rumination and subsequent mood change in dysphoric and depressed individuals (Lyubomirsky \& Nolen-Hoeksema, 1993; Morrow \& Nolen-Hoeksema, 1990). The rumination statements, which were developed to elicit self-focus related to depression-relevant constructs, are not inherently negative or positive, but are open to individual interpretation. Indeed, behavioral studies using these statements have found no effect of these statements on control participants but increases in negative mood and enhanced cognitive biases in dysphoric and depressed participants (Nolen-Hoeksema et al., 2008).

We hypothesized that while thinking about these statements, major depressive disordered (MDD) and nondepressed control (CTL) participants will activate different types of representations, memories, or self-related thoughts that will be accompanied by different patterns of neural activation. More specifically, we expected that, as compared with controls, depressed participants would exhibit greater activation in limbic regions (i.e., amygdala, insula, and parahippocampus), rACC, and MPFC during ruminative thought, reflecting increased emotional activation and retrieval of autobiographical memories.

\section{METHOD}

\section{Participants}

Fourteen individuals who were diagnosed with MDD and 14 healthy (CTL) individuals were diagnosed using the Structured Clinical Interview for the DSM-IV (First, Gibbon, Spitzer, \& Williams, 1995) and completed the Beck Depression Inventory II (BDI; Beck, Steer, \& Garbin, 1988) and the Ruminative Response Styles Questionnaire (RSQ; Nolen-Hoeksema \& Morrow, 1991) (see Table 1). MDD participants were excluded if they reported alcohol or substance abuse symptoms in the last 6 months or if they were diagnosed with current comorbid social anxiety disorder. Control participants were free of any lifetime diagnosis of an Axis I disorder and scored less than 10 on the BDI. All participants were fluent in English and free of head trauma, and were paid $\$ 25$ per hour for their participation in the study.

\section{RUM IND Task}

The RUM IND task included 10 statements from each of three conditions: rumination (RUM; "Think about what people notice about your personality"), abstract distraction (ABS; "Think about what contributes to team spirit"), and concrete distraction (CON; "Think about a row of shampoo bottles on display"). Most previous behavioral rumination studies have used the CON distraction statements (Nolen-Hoeksema \& Morrow, 1991) as a control condition. Importantly, however, these statements differ from the rumination statements on several dimensions. For example, the CON distraction statements invoke specific, often stereotypical, visually dependent images, whereas the RUM statements ask participants to generate more open-ended conceptual representations. In order to adapt the rumination versus distraction design to the scanning environment, we needed an additional control condition that would be better matched conceptually to the rumination statements. Therefore, we included a second distraction condition, ABS, to permit a contrast that, as compared with the CON statements, would be less visual and less reliant on mental representations of external objects or scenes. We hypothesized that the ABS distraction condition would allow us to isolate neural activation in a control condition that is more closely related to abstract ruminative thought. A complete list of the statements is presented in Appendix 2 of the online Supplemental Materials.

For each statement, participants were asked to focus their attention on the idea presented in the statement and to visualize and concentrate on each statement as it appeared (a total of 30 statements, each appearing in black text on the screen for $30 \mathrm{sec}$ and separated by a 10 -sec fixation cross). Participants were instructed to stop thinking about the previous statement and to clear their minds for the next statement whenever a fixation replaced a statement. Five statements (interspersed with fixation crosses) from each set were presented consecutively in a fixed order to form a block. At the end of each 
Table 1

Demographic Information for Depressed and Healthy Control Participants

\begin{tabular}{lrrrr}
\hline & MDD & $S D$ & CTL & $S D$ \\
\hline Participants & $N=14$ & & $N=14$ & \\
Sex M:F & $6: 8$ & & $5: 9$ & \\
Mean age & 40.6 & 11.3 & 34.6 & 10.4 \\
Handedness R:L & $14: 0$ & & $10: 4$ & \\
Mean education1 & 2.2 & 0.7 & 2.5 & 0.7 \\
Medications Med:NoMed ${ }^{2}$ & $6: 8$ & & N/A & \\
Lifetime number of episodes & $N=7 ; 1-6$ episodes & & N/A & \\
& $N=7 ;>10$ episodes & & & \\
Mean duration of current episode in months & 14.3 & 19.1 & N/A & \\
Mean BDI score* & 24.0 & 10.5 & 1.0 & 2.0 \\
& range: $9-45$ & & range: $0-7$ & \\
Mean RSQ score* $^{*}$ & 51.2 & 18.0 & 29.1 & 7.9 \\
& range: $23-73$ & & range: $22-38$ & \\
\hline
\end{tabular}

Note-MDD, depressed group; CTL, control group; BDI, Beck Depression Inventory; RSQ, Ruminative Response Styles questionnaire. 1Education levels based on an ordinal scale of 1 (no college) to 4 (graduate degree). ${ }^{2}$ Two MDD participants were taking venlafaxine (Effexor), the remaining 4 MDD participants were taking duloxetine (Cymbalta), escitalopram (Lexapro), fluoxetine (Prozac), and citalopram (Celexa). ${ }^{*} p<.001$.

block, a rating screen appeared for $10 \mathrm{sec}$; participants were asked to rate on a scale of 1-4 how sad they are right now, using the button box. Each scanner run consisted of a block of each of the three conditions, RUM, CON, and ABS, and three sadness ratings. Two runs were conducted for each participant, always beginning with the RUM block (in order to limit any state mood effects), and were counterbalanced so that half of the participants received Run 1 first (RUM [statements 1-5], CON [statements 1-5], and ABS [statements 1-5]), and the other half received Run 2 first (RUM [statements 6-10], CON [statements 6-10], and ABS [statements 6-10]).

\section{Imaging Analysis}

Functional scans were acquired on a 1.5T GE Signa scanner using a $\mathrm{T} 2^{*}$ in-/out- spiral pulse sequence $\left(\mathrm{TE}=40 \mathrm{msec}\right.$, flip $\left.=90^{\circ}\right)$ consisting of twenty-four 4-mm interleaved slices (axial in-plane resolution $3.75 \times 3.75 \mathrm{~mm}$, no gap) at a temporal resolution of $2 \mathrm{sec}(2.00 \mathrm{TR})$. All preprocessing and analyses were conducted using an analysis of functional neural images (AFNI; Cox, 1996). Time series data were concatenated, slice time and motion corrected, and participants who moved more than $1 \mathrm{~mm}$ were excluded. Data were spatially smoothed with a 4-mm Gaussian kernel, high-pass filtered, converted to percent signal change, and coregistered to anatomical images. Preprocessed time series data for each individual were analyzed with multiple regression. The primary contrasts (RUM vs. ABS and RUM vs. CON) were convolved with a canonical hemodynamic gamma-variate function response, including terms for residual motion, trend, and contrast regressors. Resulting individual $t$ statistic maps were transformed into $Z$ scores and were warped into Talairach space. The CTL and MDD group maps were analyzed with a two-sample $t$ test for each contrast using the AFNI software program, AlphaSim, and a joint voxelwise and cluster-size threshold was determined for a cluster threshold protected at $p<.05$. A Monte Carlo simulation with 1,000 iterations was conducted with a voxelwise threshold of $p<.005$, Gaussian kernel smoothing of $3.75 \mathrm{~mm}^{3}$ (equivalent to one original voxel), and a cluster minimum of 10 voxels. A final clusterwise threshold of $p<.02$ was determined, and a list of all clusters of activation meeting these criteria was generated.

\section{RESULTS}

\section{Behavioral Results}

A two-way (group [MDD, CTL] repeated over condition [RUM, ABS, CON]) ANOVA conducted on sad mood ratings yielded only significant main effects of group $[F(1,26)=23.72, p<.0001]$, reflecting greater sadness ratings reported overall by the MDD participants, and condition $[F(2,26)=3.86, p<.03]$, reflecting the finding that participants gave significantly greater sadness ratings following RUM versus ABS $[t(27)=3.10, p<.005]$. The interaction of group and condition was not significant $[F(2,26)=2.65, p>.05]$. A similar two-way ANOVA conducted on reaction times (RTs) for the mood ratings yielded a significant main effect for group, indicating that the MDD participants were slower to respond overall than were the CTL participants $[F(1,26)=11.07, p<.003]$; neither the main effect for condition $[F(2,26)=1.79]$ nor the interaction of group and condition $[F(2,26)=2.90$, both $p s>.05$ ] was significant (see Table 2).

\section{Imaging Results}

Imaging data for both within-group and between-groups analyses are presented for the two primary contrasts of interest: RUM versus CON and RUM versus ABS.

\section{RUM Versus CON}

Within-group activations. Within-group activations for RUM versus CON are listed in Supplemental Appendix 1, Table 1A (MDD) and Table 1B (CTL). For the RUM $>$ CON contrast, within the MDD group, significant activations were present in the rACC (BA 32), the inferior parietal lobule, insula, middle frontal and occipital gyri, precentral gyrus, precuneus, superior temporal gyrus, and thalamus. The CTL group exhibited activations in the inferior and superior parietal lobules, precuneus, pre- and postcentral gyri, the fusiform gyrus, middle occipital gyrus, and the superior frontal gyrus.

Between-groups activations. Table 3 presents between-groups activations for the RUM $>\mathrm{CON}$ contrast. As compared with the MDD participants, CTL participants exhibited significantly stronger activations in the caudate and visual association regions, including 
Table 2

Mood Ratings and Reaction Times (RTs, in Milliseconds) for Each Condition

\begin{tabular}{|c|c|c|c|c|c|c|}
\hline \multirow[b]{2}{*}{ Group } & \multicolumn{2}{|c|}{ RUM (Sad) } & \multicolumn{2}{|c|}{$\mathrm{CON}$ (Sad) } & \multicolumn{2}{|c|}{ ABS (Sad) } \\
\hline & $M$ & $S D$ & $M$ & $S D$ & $M$ & $S D$ \\
\hline \multicolumn{7}{|c|}{ Mood Ratings } \\
\hline CTL & 1.11 & 0.21 & 1.14 & 0.53 & 1.00 & 0.00 \\
\hline MDD & 2.61 & 1.21 & 2.07 & 0.81 & 1.93 & 0.96 \\
\hline \multicolumn{7}{|c|}{ RTs } \\
\hline CTL & $1,889.61$ & 719.14 & $1,667.86$ & 604.59 & $2,031.89$ & $1,382.37$ \\
\hline MDD & $3,531.04$ & $1,422.15$ & $2,965.00$ & $1,737.34$ & $2,270.18$ & $1,260.50$ \\
\hline
\end{tabular}

Note-CTL, control group; MDD, depressed group; RUM, rumination; CON, concrete distraction; $\mathrm{ABS}$, abstract distraction.

the lingual gyrus, cuneus, precuneus, and fusiform gyrus. MDD participants exhibited greater activations than did CTL participants in the thalamus, middle frontal gyrus (BA 9; DLPFC), orbital frontal gyrus (BA 11; OFC), and subgenual anterior cingulate (BA 25; sgACC).

\section{RUM Versus ABS}

Within-group activations. Within-group activations for RUM versus ABS are listed in the online Supplemental Appendix 1, Table 2A (MDD) and Table 2B (CTL). MDD participants exhibited significant activations in the RUM $>$ ABS contrast within the dorsal anterior cingulate cortex (BA 32; dACC), cuneus, inferior frontal gyrus, inferior parietal lobule, medial frontal gyrus (BA 10; MPFC), and middle occipital and temporal gyri. CTL participants exhibited activations in the cuneus and precuneus, medial frontal gyrus (BA 10; MPFC), and superior frontal gyrus (BA 9; DLPFC).

Between-groups activations. Significant betweengroups activations for the RUM versus ABS contrast are listed in Table 4. In response to the RUM $>$ ABS contrast, CTL participants had greater activation than did MDDs in only the inferior frontal gyrus (BA 9; DLPFC). In contrast, the MDD participants had significantly greater activation than did the CTL participants in the dACC (BA 24), rACC (BA 32), inferior parietal lobule, middle frontal gyrus
(BA 46; DLPFC), middle occipital gyrus, parahippocampus and amygdala, posterior cingulate cortex (BA 31; PCC), and middle and superior temporal gyri.

\section{DISCUSSION}

The present study used fMRI to examine patterns of neural activation as depressed individuals ruminate. Because rumination involves a number of specific cognitive and affective processes, including self-focused attention, self-referential processing, and recall of autobiographical memories, we hypothesized that MDD participants would exhibit increased activation during rumination in regions associated with emotional processing (e.g., amygdala), retrieval of autobiographical memory (e.g., parahippocampus), and the processing of self-related concepts (e.g., MPFC). In addition to including a distraction condition with concrete statements that have been used in previous behavioral studies of rumination, we added a novel set of abstract distraction statements that we hypothesized would better isolate differential activation due to emotional processing, memory retrieval, and self-focus during rumination. Supporting this prediction, the two contrasts, RUM versus CON and RUM versus ABS, highlight different patterns of activation associated with rumination.

Table 3

Significant Activations Between Depressed (MDD) and Control (CTL) Groups for Rumination Versus Concrete Distraction Contrast

\begin{tabular}{|c|c|c|c|c|c|c|c|}
\hline \multirow[b]{2}{*}{ Region } & \multirow[b]{2}{*}{ Side } & \multirow[b]{2}{*}{ BA } & \multicolumn{3}{|c|}{ Talairach Coord. } & \multirow[b]{2}{*}{ Voxels } & \multirow[b]{2}{*}{$\operatorname{Max} Z$} \\
\hline & & & $x$ & $y$ & $z$ & & \\
\hline \multicolumn{8}{|l|}{ CTLs $>$ MDDs } \\
\hline Caudate & $\mathrm{L}$ & & -8 & 19 & 12 & 26 & -3.10 \\
\hline Cuneus & Bilat & & -8 & -86 & 38 & 80 & -3.14 \\
\hline Fusiform gyrus & $\mathrm{L}$ & & -30 & -56 & -14 & 19 & -2.72 \\
\hline Lingual gyrus & $\mathrm{L}$ & & -4 & -83 & 1 & 178 & -3.64 \\
\hline Precuneus & Bilat & 7 & 0 & -75 & 57 & 16 & -2.84 \\
\hline \multicolumn{8}{|l|}{ MDDs $>$ CTLs } \\
\hline Middle frontal gyrus (DLPFC) & $\mathrm{L}$ & $9 / 47$ & -34 & 11 & 31 & 17 & 3.37 \\
\hline Orbital frontal gyrus (OFC) & $\mathrm{R}$ & 11 & 4 & 30 & -26 & 17 & 3.29 \\
\hline Anterior cingulate (sgACC) & $\mathrm{L}$ & 25 & -4 & 15 & -7 & 13 & 2.65 \\
\hline Thalamus & $\mathrm{R}$ & & 4 & -26 & 4 & 11 & 2.77 \\
\hline
\end{tabular}

Note-DLPFC, dorsolateral prefrontal cortex; OFC, orbitofrontal cortex; sgACC, subgenual anterior cingulate cortex. Side indicates right $(\mathrm{R})$ or left $(\mathrm{L})$ of midline or bilateral (Bilat). BA refers to corresponding Brodmann area. Talairach coordinates reported in RAI $(x=$ right, $y=$ anterior, $z=$ inferior) convention. Max $Z$ value represents the $Z$ score at the maximum voxel within a cluster. Cluster threshold $>10$ voxels; voxelwise $p<.005$; clusterwise $p<.02$, corrected. 
Table 4

Significant Activations Between Depressed (MDD) and Control (CTL) Groups for Rumination Versus Abstract Distraction Contrast

\begin{tabular}{|c|c|c|c|c|c|c|c|}
\hline \multirow[b]{2}{*}{ Region } & \multirow[b]{2}{*}{ Side } & \multirow[b]{2}{*}{ BA } & \multicolumn{3}{|c|}{ Talairach Coord. } & \multirow[b]{2}{*}{ Voxels } & \multirow[b]{2}{*}{$\operatorname{Max} Z$} \\
\hline & & & $x$ & $y$ & $z$ & & \\
\hline \multicolumn{8}{|l|}{ CTLs $>$ MDDs } \\
\hline Inferior frontal gyrus (DLPFC) & $\mathrm{R}$ & 9 & 60 & 8 & 31 & 11 & 3.01 \\
\hline \multicolumn{8}{|l|}{ MDDs $>$ CTLs } \\
\hline Anterior cingulate (dACC) & $\mathrm{L}$ & 24 & -19 & -15 & 34 & 17 & 3.14 \\
\hline Anterior cingulate (rACC) & Bilat & 32 & -8 & 11 & 46 & 16 & 3.67 \\
\hline Inferior parietal lobule & Bilat & 40 & -30 & -45 & 34 & 22 & 2.89 \\
\hline Middle frontal gyrus (DLPFC) & Bilat & 46 & -56 & 34 & 16 & 17 & 2.81 \\
\hline Middle occipital gyrus & $\mathrm{R}$ & 19 & 26 & -83 & 19 & 15 & 2.65 \\
\hline Middle temporal gyrus & Bilat & 21 & -64 & -26 & -7 & 34 & 3.43 \\
\hline Parahippocampus and amygdala & $\mathrm{R}$ & 28 & 19 & -8 & -11 & 13 & 3.04 \\
\hline Posterior cingulate cortex (PCC) & Bilat & 31 & 15 & -38 & 34 & 18 & 3.60 \\
\hline Superior temporal gyrus & $\mathrm{L}$ & 22 & -56 & 8 & -3 & 13 & 2.73 \\
\hline
\end{tabular}

Note-DLPFC, dorsolateral prefrontal cortex; dACC, dorsal anterior cingulate cortex; rACC, rostral anterior cingulate cortex; PCC, posterior cingulate cortex. Side indicates right (R) or left (L) of midline or bilateral (Bilat). BA refers to corresponding Brodmann area. Talairach coordinates reported in RAI ( $x=$ right, $y=$ anterior, $z=$ inferior) convention. Max $Z$ value represents the $Z$ score at the maximum voxel within a cluster. Cluster threshold $>10$ voxels; voxelwise $p<.005$; clusterwise $p<.02$, corrected.

Within-group analyses of the RUM versus CON contrast reveal activations common to both the depressed and control groups in regions affiliated with higher order visual association and attention such as the middle occipital gyrus, precuneus, and inferior parietal lobule. Betweengroup analyses for RUM versus CON indicate greater activation for CTLs compared with MDDs in regions associated with the generation of mental imagery, including the cuneus, precuneus, fusiform gyrus, and lingual gyrus; in contrast, MDD participants exhibited greater activation in regions associated with the generation and regulation of mood states: sgACC, OFC, thalamus, and DLPC.

The increased activation observed in the sgACC in the RUM versus CON contrast is of particular interest. The sgACC has garnered considerable attention both for its putative role in a mood-regulation circuit and for its specific role in models of depression (Drevets, 2000; Gotlib \& Hamilton, 2008; Mayberg et al., 1999). In the context of the present study, it is possible that activation in the sgACC reflects autonomic aspects of emotional processing during the RUM condition as compared with the CON condition. The sgACC, in tandem with other ventral regions such as the $\mathrm{OFC}$, is proposed to integrate multiple domains of the experience of emotion, including autonomic functioning (Critchley, 2005). Thus, the sgACC activation observed in depressed individuals may indicate that they are responding to rumination statements with a greater degree of emotion than are controls. Although it is interesting that the RUM condition was associated with greater activation in a prominent region in neural models of depression (i.e., sgACC) than was the CON condition, it is not clear that this contrast disambiguates neural differences specific to the cognitive and affective subprocesses that characterize rumination.

Thus, we developed a second contrast, RUM versus ABS, to assess more directly depression-associated differences during rumination as compared with representing more abstract and complex concepts. Consistent with findings of previous investigations of self-focus, the within-group analyses of the RUM versus ABS contrast yielded activations in the MPFC (BA 10) in both MDDs and CTLs. Moreover, the between-groups comparison of activations during the RUM versus ABS conditions revealed robust differences in hypothesized regions, with MDD participants exhibiting greater activation than did CTLs in the amygdala, parahippocampus, rACC, MPFC, and posterior cingulate.

The amygdala has been the focus of numerous investigations of depression (e.g., Drevets, 2003; see Gotlib \& Hamilton, 2008, for a review). In particular, Siegle et al. (2006) and Siegle et al. (2002) reported sustained amygdala activation to be correlated with self-reported rumination. These data, however, represent the first evidence that the amygdala is indeed differentially active in depressed than in nondepressed individuals during rumination.

We also found greater rACC activation in the MDDs than in the CTLs during RUM versus ABS. The rACC-a subregion of the MPFC - has frequently been implicated in depression (e.g., Eugène, Joormann, Cooney, Atlas, \& Gotlib, 2010; Steele, Currie, Lawrie, \& Reid, 2007). Investigators have found increased activation in $\mathrm{rACC}$ in healthy participants as they are attending to subjective feeling states (e.g., Lane, Fink, Chau, \& Dolan, 1997). The rACC activation in the present study may reflect increased attention toward one's internal state that is unique to the depressed participants. Increased self-focus or attention toward those emotional responses may, in turn, bolster the higher level of recruitment of rACC that we observed in the MDD participants.

As compared with the controls, depressed participants also exhibited increased activation in the PCC in the RUM versus ABS contrast. This structure has been highlighted as part of the "default mode" network (Greicius, Krasnow, Reiss, \& Menon, 2003; Raichle, 2001) and is hypothesized to be active during the generation of internal 


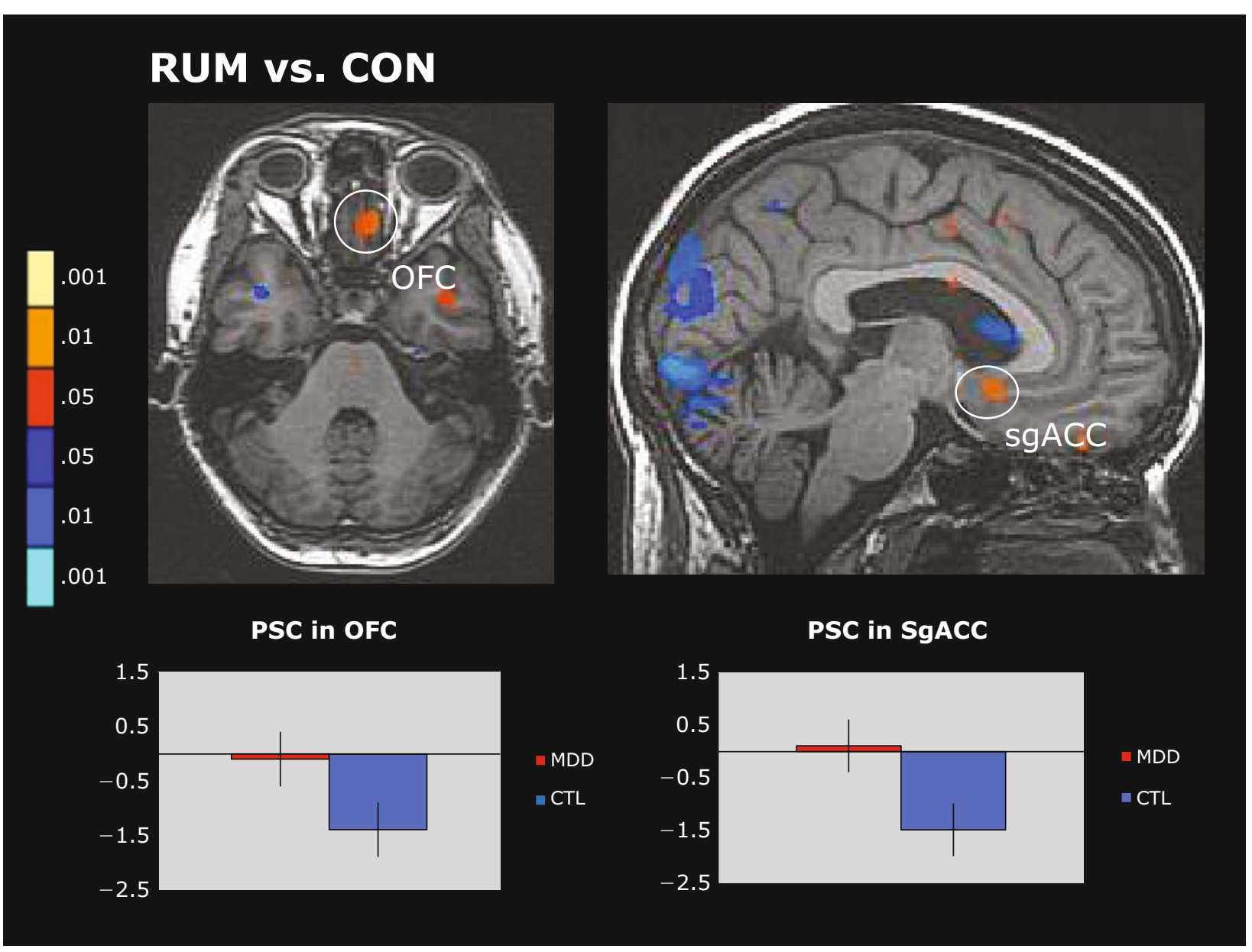

Figure 1. Activations to rumination (RUM) versus concrete $(\mathrm{CON})$ distraction contrast. Yellow activations, MDD > CTL; blue activations, CTL $<$ MDD. Left $=$ left. Orbitofrontal cortex $(4,30,-26)$. Subgenual anterior cingulate $(B A 25 ;-4,15,-7), p<.02$, corrected. MDD, depressed group; CTL, control group.

thought and inhibited during various cognitive tasks. Interestingly, the rumination condition used in the present study is similar to some resting-state conditions used in investigations of the default mode network. Within this network, anterior regions are hypothesized to be associated with self-focused aspects of internal thoughts; posterior regions, including the PCC, are associated with the retrieval of autobiographical memories. The increased activation within the parahippocampus also observed in the MDD group suggests that during RUM versus ABS, they are engaging in more mood-congruent memory retrieval. Importantly, CTL participants also exhibited activation within the PCC during the RUM versus ABS contrast, but did so to a lesser extent than did MDDs. This finding supports recent observations that depressed individuals are characterized by impaired context-dependent reductions in activation in default-mode regions (Grimm et al., 2009; Sheline et al., 2009).

A final region of activation that we should consider here, common to both depressed and healthy control participants, is the DLPFC. The RUM versus CON contrast identified greater activation in DLPFC in MDDs than in
CTLs. In response to RUM versus ABS, both MDDs and CTLs exhibited activation in different subregions of the DLPFC (BA 46 and BA 9, respectively). Our findings are consistent with work demonstrating greater activation in DLPFC during self-referential processing in depressed individuals (Lemogne et al., 2009), and with the growing literature documenting structural and functional irregularities in the DLPFC in depression (e.g., Fitzgerald et al., 2006; Koenigs et al., 2008). Considered in light of the strong association between rumination and the development and maintenance of depressed mood, the differential pattern of activation observed in DLPFC in MDD as compared with that in CTL participants (i.e., increased DLPFC activation in MDDs during rumination as compared with both the abstract and concrete distraction conditions) reinforces the importance of continued investigation of this region. At this early stage, it is not yet clear whether hyperactivation of the DLPFC during rumination in depression reflects an impaired regulatory mechanism or whether the DLPFC may indicate recruitment toward more basic cognitive demands during self-focus - a question that is important for future research to address. 


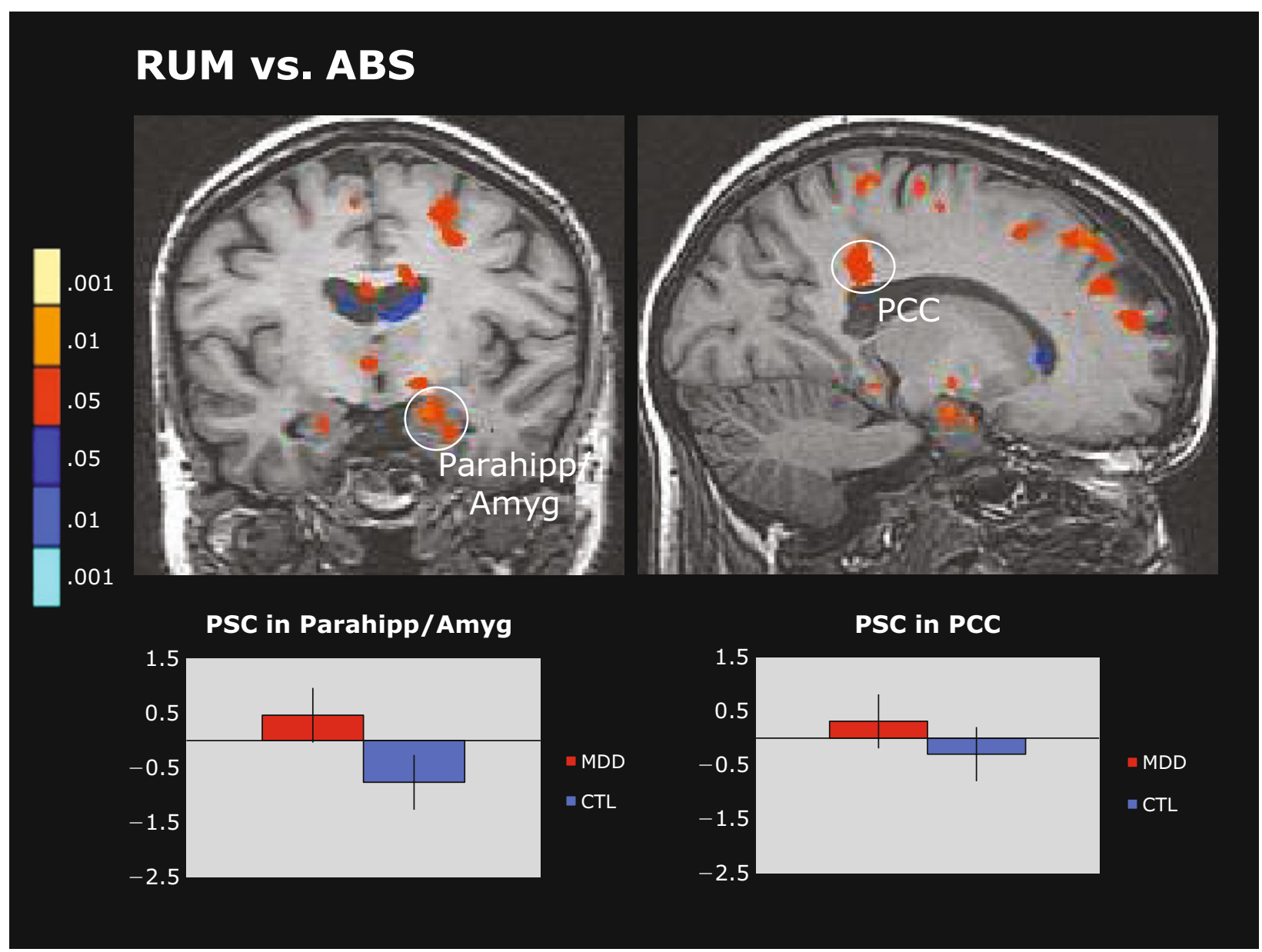

Figure 2. Activations to rumination (RUM) versus abstract (ABS) distraction contrast. Yellow activations, MDD > CTL; blue activations, CTL < MDD. Left = left. Posterior cingulate cortex $(15,-38,34)$. Parahippocampus/amygdala $(19,-8,-11) ; p<.02$, corrected. MDD, depressed group; CTL, control group.

It is important to note a number of limitations of the present study. For example, to emulate the manner in which rumination has been induced behaviorally, we used a modified block design to give participants a relatively long, consistent period of time during which to focus on the various statements. In future studies, investigators might consider using other approaches, such as eventrelated designs that alternate between various conditions, or that vary the length of presentation of the statements or the duration of the intervening fixation periods. We should note that we presented only neutral statements in the present study. Additional research examining neural functioning in depressed and healthy control individuals in response to positive and negative stimuli is necessary to explore systematically group differences in ruminative self-focus induced by valenced material. It is also possible that some of the activation we observed was due to state effects of sadness; that is, MDD and CTL participants differed in baseline mood prior to being scanned, as well as in mood during the scan. In addition to investigating the effects of transient sad mood in healthy controls, future work examining high- versus low-ruminating depressed individuals may help to clarify whether the neural profile of depressed individuals during ruminative self-focus is unique to this disorder. Finally, given the complexity of rumination, as well as the exploratory nature of the task, it is important for us to caution that our inferences regarding associations between activation and specific subprocesses are necessarily speculative.

The present findings contribute to our understanding of the basis of rumination in depression by elucidating the neural regions that are tonically overactive in depression and those that are activated by specific types of tasks. By investigating the neural basis of rumination as a mechanism by which negative mood is enhanced, we gain insight into the cognitive processes that characterize depressed individuals as they ruminate, allowing us to create tasks to interrogate more effectively neural aspects of depression. Most importantly, greater understanding of these neural patterns will allow us to refine our ability to diagnose and treat individuals diagnosed with MDD. 


\section{AUTHOR NOTE}

This research was supported by National Institute of Mental Health Grant MH59259 awarded to I.H.G. We thank Lauren Y. Atlas and Hannah S. Kang for their assistance in data collection and technical support. The authors report no biomedical financial interests or potential conflicts of interest. Address correspondence to R. E. Cooney, Department of Psychology, Stanford University, Bldg. 420, Jordan Hall, Stanford, CA 94305 (e-mail: rcooney@stanford.edu).

\section{REFERENCES}

Beck, A. T., Steer, R. A., \& Garbin, M. G. (1988). Psychometric properties of the Beck Depression Inventory: Twenty-five years of evaluation. Clinical Psychology Review, 8, 77-100. doi:10.1016/0272 -7358(88)90050-5

Cox, R. W. (1996). AFNI, software for analysis and visualization of functional magnetic resonance neuroimages. Computers \& Biomedical Research, 29, 162-173.

CRITCHLEy, H. D. (2005). Neural mechanisms of autonomic, affective, and cognitive integration. Journal of Comparative Neurology, 493, 154-166. doi:10.1002/cne. 20749

DREVETS, W. C. (2000). Neuroimaging studies of mood disorders. Biological Psychiatry, 48, 813-829. doi:10.1016/S0006-3223(00)01020-9

DREVETS, W. C. (2003). Neuroimaging abnormalities in the amygdala in mood disorders. In P. Shinnick-Gallagher, A. Pitkänen, A. Shekhar, \& L. Cahill (Eds.), The amygdala in brain function: Basic and clinical approaches (Vol. 985, pp. 420-444). New York: New York Academy of Sciences. doi:10.1111/j.1749-6632.2003.tb07098.x

EugÈne, F., Joormann, J., Cooney, R. E., Atlas, L. Y., \& Gotlib, I. H. (2010). Neural correlates of inhibitory deficits in depression. Psychiatry Research: Neuroimaging, 181, 30-35. doi:10.1016/j .pscychresns.2009.07.010

First, M. B., Gibbon, M., Spitzer, R. L., \& Williams, J. B. W. (1995). User's guide for the structured clinical interview for DSM-IV Axis I disorders: SCID-I (Version 2.0). Arlington, VA: American Psychiatric Publishing.

Fitzgerald, P. B., Oxley, T. J., Laird, A. R., Kulkarni, J., Egan, G. F., \& Daskalakis, Z. J. (2006). An analysis of functional neuroimaging studies of dorsolateral prefrontal cortical activity in depression. Psychiatry Research, 148, 33-45. doi:10.1016/j.pscychresns .2006 .04 .006

Fossati, P., Hevenor, S. J., Graham, S. J., Grady, C., Keightley, M. L., Craik, F., \& MAYberg, H. (2003). In search of the emotional self: An fMRI study using positive and negative emotional words. American Journal of Psychiatry, 160, 1938-1945.

Gotlib, I. H., \& Hamilton, J. P. (2008). Neuroimaging and depression: Current status and unresolved issues. Current Directions in Psychological Science, 17, 159-163. doi:10.1111/j.1467-8721.2008.00567.x

Gotlib, I. H., \& JoormanN, J. (2010). Cognition and depression: Current status and future directions. Annual Review of Clinical Psychology, 6, 285-312. doi:10.1146/annurev.clinpsy.121208.131305

Greicius, M. D., Krasnow, B., Reiss, A. L., \& Menon, V. (2003). Functional connectivity in the resting brain: A network analysis of the default mode hypothesis. Proceedings of the National Academy of Sciences, 100, 253-258. doi:10.1073/pnas.0135058100

Grimm, S., Ernst, J., Boesiger, P., Schuepbach, D., Hell, D., BOEKER, H., \& Northoff, G. (2009). Increased self-focus in major depressive disorder is related to neural abnormalities in subcorticalcortical midline structures. Human Brain Mapping, 30, 2617-2627. doi:10.1002/hbm.20693

Hamilton, J. P., \& Gotlib, I. H. (2008). Neural substrates of increased memory sensitivity for negative stimuli in major depression. Biological Psychiatry, 63, 1155-1162. doi:10.1016/j.biopsych.2007 .12 .015

Howe, M. L., \& Courage, M. L. (1997). The emergence and early development of autobiographical memory. Psychological Review, 104, 499-523.

Johnson, M. K., Nolen-Hoeksema, S., Mitchell, K. J., \& Levin, Y. (2009). Medial cortex activity, self-reflection and depression. Social Cognitive \& Affective Neuroscience, 4, 313-327. doi:10.1093/scan/ nsp022
Johnson, M. K., RaYe, C. L., Mitchell, K. J., Touryan, S. R., Greene, E. J., \& Nolen-Hoeksema, S. (2006). Dissociating medial frontal and posterior cingulate activity during self-reflection. Social Cognitive \& Affective Neuroscience, 1, 56-64. doi:10.1093/scan/ns1004

Joormann, J., Dkane, M., \& Gotlib, I. H. (2006). Adaptive and maladaptive components of rumination? Diagnostic specificity and relation to depressive biases. Behavior Therapy, 37, 269-280. doi:10.1016/j.beth.2006.01.002

Keedwell, P. A., Andrew, C., Williams, S. C. R., Brammer, M. J., \& PhILlips, M. L. (2005). A double dissociation of ventromedial prefrontal cortical responses to sad and happy stimuli in depressed and healthy individuals. Biological Psychiatry, 58, 495-503. doi:10.1016/j biopsych.2005.04.035

Koenigs, M., Huey, E. D., Calamia, M., Raymont, V., Tranel, D., \& GRAFMAN, J. (2008). Distinct regions of prefrontal cortex mediate resistance and vulnerability to depression. Journal of Neuroscience, 28, 12341-12348. doi:10.1523/JNEUROSCI.2324-08.2008

Lane, R. D., Fink, G. R., Chau, P. M., \& Dolan, R. J. (1997). Neural activation during selective attention to subjective emotional responses. NeuroReport, 8, 3969-3972.

Lemogne, C., le Bastard, G., Mayberg, H., Volle, E., BergouiGNAN, L., LEHÉRICY, S., ET AL. (2009). In search of the depressive self: Extended medial prefrontal network during self-referential processing in major depression. Social Cognitive \& Affective Neuroscience, 4, 305-312. doi:10.1093/scan/nsp008

Lyubomirsky, S., \& Nolen-Hoeksema, S. (1993). Self-perpetuating properties of dysphoric rumination. Journal of Personality \& Social Psychology, 65, 339-349.

MAGUIRE, E. A. (2001). Neuroimaging studies of autobiographical event memory. Philosophical Transactions of the Royal Society B, 356, 1441-1451. doi:10.1098.rstb.2001.0944

Mayberg, H. S., Liotti, M., Brannan, S. K., McGinnis, S., MahURIN, R. K., JerabeK, P. A., Et AL. (1999). Reciprocal limbiccortical function and negative mood: Converging PET findings in depression and normal sadness. American Journal of Psychiatry, 156, 675-682.

Morrow, J., \& Nolen-Hoeksema, S. (1990). The effects of response styles for depression on the remediation of depressive affect. Journal of Personality \& Social Psychology, 58, 519-527.

Nolen-Hoeksema, S., \& Morrow, J. (1991). A prospective study of depression and posttraumatic stress symptoms after a natural disaster: The 1989 Loma Prieta earthquake. Journal of Personality \& Social Psychology, 61, 115-121.

Nolen-Hoeksema, S., \& Morrow, J. (1993). Effects of rumination and distraction on naturally-occurring depressed mood. Cognition \& Emotion, 7, 561-570.

Nolen-Hoeksema, S., Wisco, B. E., \& Lyubomirsky, S. (2008). Rethinking rumination. Perspectives on Psychological Science, 3, 400424. doi:10.1111/j.1745-6924.2008.00088.x

OChSNer, K., \& Gross, J. J. (2005). The cognitive control of emotion. Trends in Cognitive Sciences, 9, 242-249. doi:10.1016/j tics.2005.03.010

Papageorgiou, C., \& Wells, A. (1999). Process and meta-cognitive dimensions of depressive and anxious thoughts and relationships with emotional intensity. Clinical Psychology \& Psychotherapy, 6, 156162. doi:10.1002/(SICI)1099-0879(199905)

Raichle, M. E. (2001). Cognitive neuroscience: Bold insights. Nature, 412, 128-130. doi: $10.1038 / 35084300$

Ray, R. D., Oschsner, K. N., Cooper, J. C., Robertson, E. R., GaBRIELI, J. D. E., \& Gross, J. J. (2005). Individual differences in trait rumination and the neural systems supporting cognitive reappraisal. Cognitive, Affective, \& Behavioral Neuroscience, 5, 156-168. doi:10.3758/CABN.5.2.156

Roberts, J., Gilboa, E., \& Gotlib, I. H. (1998). Ruminative response style and vulnerability to depressive episodes: Factor components, mediating processes, and episode duration. Cognitive Therapy \& Research, 22, 401-425.

Schmitz, T. W., \& Johnson, S. C. (2006). Self-appraisal decisions evoke dissociated dorsal-ventral aMPFC networks. NeuroImage, 30, 1050-1058. doi:10.1016/j.neuroimage.2005.10.030

Segerstrom, S. C., Tsao, J. C. I., Alden, L. E., \& Craske, M. G. (2000). Worry and rumination: Repetitive thought as a concomitant 
and predictor of negative mood. Cognitive Therapy \& Research, 24, 671-688. doi:10.1023/A:1005587311498

Sheline, Y. I., Barch, D. M., Price, J. L., Rundle, M. M., VaishNAVI, S. N., SNYDER, A. Z., ET AL. (2009). The default mode network and self-referential processes in depression. Proceedings of the National Academy of Sciences, 106, 1942-1947. doi:10.1073/ pnas. 0812686106

Siegle, G. J., Carter, C. S., \& Thase, M. E. (2006). Use of fMRI to predict recovery from unipolar depression with cognitive behavior therapy. American Journal of Psychiatry, 163, 735-738. doi:10.1176/ appi.ajp.163.4.735

Siegle, G. J., Steinhauer, S. R., Thase, M. E., Stenger, V. A., \& CARTER, C. S. (2002). Can't shake that feeling: fMRI assessment of sustained amygdala activity in response to emotional information in depressed individuals. Biological Psychiatry, 51, 693-707.

Steele, J. D., Currie, J., LaWrie, S. M., \& Reid, I. (2007). Prefrontal cortical functional abnormality in major depressive disorder: A stereotactic meta-analysis. Journal of Affective Disorders, 101, 1-11. doi:10.1016/j.jad.2006.11.009
Svoboda, E., McKinnon, M. C., \& Levine, B. (2006). The functional neuroanatomy of autobiographical memory: A meta-analysis. Neuropsychologia, 44, 2189-2208. doi:10.1016/j.neuropsychologia 2006.05.023

Watkins, E., Moulds, M., \& Mackintosh, B. (2005). Comparisons between rumination and worry in a non-clinical population. Behaviour Research \& Therapy, 43, 1577-1585. doi:10.1016/j.brat 2004.11.008

\section{SUPPLEMENTAL MATERIALS}

The significant activations within the depressed and control groups and the concrete and abstract rumination statements we presented to the participants may be downloaded from http://cabn.psychonomic-journals .org/content/supplemental.

(Manuscript received January 26, 2010; revision accepted for publication August 5, 2010.) 